\title{
Trade liberalization and the allocation over domestic and foreign supplies: a case study for Spanish manufacturing *
}

\author{
P.M.C. de Boer $\stackrel{\dagger}{\dagger}$ C. Martinez $\underset{\ddagger}{\ddagger}$ R. Harkema ${ }^{\S}$
}

ECONOMETRIC INSTITUTE REPORT EI9829/A

\begin{abstract}
The purpose of the present paper is to investigate whether Spain's accession to the European Union in 1986 caused a structural break in the allocation of total supplies of manufactures over domestic and foreign supplies. To that end we use the homogeneity-constrained Almost Ideal Demand System to specify the long-run equilibrium relationships between the shares in total supplies and total real demand and relative prices and a first-order error correction mechanism in order to describe the adjustment process to equilibrium. Using a formal statistical test, it turns out that a structural break actually occurred and led to a rather sharp decrease in the share of Spain and an increase in the shares of the other members of the European Union.
\end{abstract}

Keywords: economic integration, structural break, impact assessment.

${ }^{*}$ The authors are indebted to Arjan Soede and Bart Hobijn for carrying out the calculations reported upon in this paper.

${ }^{\dagger}$ Econometric Institute, Erasmus University Rotterdam, P.O.Box 1738, 3000 DR Rotterdam, The Netherlands, e-mail: pmdeboer@few.eur.nl

${ }^{\ddagger}$ European Commission. Views expressed in this paper are those of the authors and should not be attributed to the European Commission. This work has been done in a private capacity.

${ }^{\S}$ Econometric Institute, Erasmus University Rotterdam, P.O.Box 1738, 3000 DR Rotterdam, The Netherlands 


\section{Contents}

\section{Introduction}

One of Spain's main economic problems since its accession to the European Union(EU) in 1986 is the sharp increase in its trade deficit. In 1984, for instance, the deficit amounted to $3.5 \%$ of gross domestic product as opposed to $6.1 \%$ in 1992 . This increase is mainly due to the increase of the trade deficit with respect to manufactures that changed from an average surplus of $0.9 \%$ over the period 1979-1985 to an average deficit of 3.8\% over the period 1986-1992. Exports of manufactures remained constant (an average of 9.8\% over both 1979-1985 and 1986-1992), whereas imports sharply rose from $8.9 \%$ over $1979-1985$ to $13.6 \%$ over 1986 -1992. Hence, the main reason of the sharp increase in Spain's trade deficit is the sharp increase in imports of manufactures. Possible cyclical explanations for this phenomenon could be the spectacular increase in total real demand and the large differences between the Spanish inflation rate and the inflation rates of the importing countries. Actually, these differences have never been so large since the sixties. A possible structural explanation could that Spain's accession to the EU led to a structural break.

The purpose of the present paper is to investigate whether Spain's accession to the EU caused a structural break in the allocation of total supplies of manufactures over domestic and foreign supplies. To that end we adopt the homogeneity- constrained Almost Ideal Demand System to specify the longrun equilibrium relationships between the shares in total supplies and total real demand and relative prices. The observed values of the shares, however, will deviate from those as specified by the long-run equilibrium relationships. This is not only due to random disturbances, but also to the occurrence of informational and reactional delays in the adaptation of the shares to changes in total real demand and relative prices. Informational delays, for example, arise because the current values of the shares are at least partly based on expectations about total real demand and relative prices instead of their actual values. Reactional delays may arise because of decisions that have been taken in previous periods. The presence of delivery lags, for example, may impede the instantaneous adjustment of the current values of the shares to their optimal values. In order to provide for an adequate explanation of the data, the specification of the set of long-run equilibrium relationships should therefore be supplemented with a dynamic specification which describes the adjustment process to equilibrium. To that purpose we shall adopt a very simple form of a general first-order error correction mechanism.

In order to test for structural break, we estimate the model using annual data which relate to the pre-integration period (in the present case 1964-1985). The test for structural break is then based on the differences between the realized values of the shares in the post-integration period (in the present case 1986-1992) and the values that the model predicts for that period. Actually, it turns out that there is strong evidence in favour of the occurrence of a structural break, making it interesting to assess the impact of the break on the shares of Spain and its trading partners in total supplies. To that end two approaches can be pursued, viz. the analytical method and the method of residual imputation. The former approach estimates separate models for the pre-integration and post-integration periods and assesses the impact by taking the differences between the shares that are predicted for the post-integration period by the pre-integration model and the estimated shares that result from the post-integration model. The latter approach assesses the impact by taking the differences between the realized shares in the post-integration period and the shares that are predicted for the post-integration period by the pre-integration model. In this paper we shall use the method of residual imputation because the number of observations is too small to estimate a separate model for the post-integration period. 
The plan of the paper is as follows. In Section 2 we present the homogeneity -constrained Almost Ideal Demand System which is used to describe the long-run equilibrium relationships between the shares in total supplies and total real demand and relative prices, we elaborate on the dynamic specification of the model, and we discuss a parsimonious specification of the covariance matrix of the error terms which is helpful in view of the limited amount of data on which the estimation procedure has to be based. Section 3 briefly reviews the estimation procedure, presents the criterion that has been adopted to select the preferred model and describes the test for structural break that has been applied. Section 4 elaborates on the data that has been used in the estimation procedure, presents the estimation results and the outcome of the test for structural break for the preferred model, and quantifies the impact of the break on the shares of Spain and its trading partners in total supplies. Section 5, finally, contains our conclusions.

\section{Specification of the model}

\subsection{The Almost Ideal Demand System}

As said before, we shall use the homogeneity-constrained Almost Ideal Demand (AID)-System as introduced by Deaton and Muellbauer (1980) in order to describe the long-run equilibrium relationships between the shares in total supplies and total real demand and relative prices. Arranging the shares in such a way that the last one represents domestic supplies, the AID-System reads as follows:

$$
\left[\begin{array}{c}
\tilde{w}_{t, 1} \\
\vdots \\
\tilde{w}_{t, n-1} \\
\tilde{w}_{t, n}
\end{array}\right]=\left[\begin{array}{lllll}
\alpha_{1} & \beta_{1} & \gamma_{1,1} & \cdots & \gamma_{1, n-1} \\
\vdots & \vdots & \vdots & & \vdots \\
\alpha_{n-1} & \beta_{n-1} & \gamma_{n-1,1} & \cdots & \gamma_{n-1, n-1} \\
\alpha_{n} & \beta_{n} & \gamma_{n, 1} & \cdots & \gamma_{n, n-1}
\end{array}\right]\left[\begin{array}{c}
1 \\
\ln \left(M_{t} / \tilde{P}_{t}\right) \\
\ln \left(p_{t, 1} / p_{t, n}\right) \\
\vdots \\
\ln \left(p_{t, n-1} / p_{t, n}\right)
\end{array}\right]
$$

where $\tilde{w}_{t, i}(i=1, \cdots, n-1 ; t=1, \cdots, T)$ denotes the equilibrium share of imports from country $i$ in total supplies at time $t, \tilde{w}_{t, n}$ represents the equilibrium share of domestic supplies in total supplies, $M_{t}$ denotes total supplies which are supposed to be equal to total demand, $p_{t, j}(j=1, \cdots, n-1)$ is the price of imports from country $j, p_{t, n}$ is the domestic price, and $\tilde{P}_{t}$ represents the Stone index, i.e., $\tilde{P}_{t}=\exp \left\{\sum_{j=1}^{n} \tilde{w}_{t, j} \ln p_{t, j}\right\}$. In obvious matrix notation (1) may be written as

$$
\tilde{w}_{t}=B \tilde{x}_{t} \quad t=1, \cdots, T
$$

The additivity constraint on the shares implies

$$
\sum_{i=1}^{n} \alpha_{i}=1 \quad \sum_{i=1}^{n} \beta_{i}=0 \quad \sum_{i=1}^{n} \gamma_{i j}=0 \quad j=1, \cdots, n-1
$$

In addition, we shall also consider the constraint of symmetry with respect to the Slutsky matrix which implies

$$
\gamma_{i j}=\gamma_{j i} \quad i, j=1, \cdots, n-1 ; i \neq j
$$

\subsection{Dynamic adjustment processes}

Because of the reasons put forward in the Introduction, the AID-System as specified in (2) has to be supplemented with a dynamic specification which describes the adjustment process to equilibrium. To that purpose we shall use the first-order error correction mechanism (ECM) as introduced by Davidson, 
Hendry, Srba and Yeo (1978). Letting $w_{t}$ denote the vector of actual shares in total supplies, the ECM reads as follows:

$$
w_{t}-w_{t-1}=\Phi\left(\tilde{w}_{t}-\tilde{w}_{t-1}\right)+\Psi\left(\tilde{w}_{t-1}-w_{t-1}\right)
$$

where $\Phi$ and $\Psi$ represent matrices of lag parameters. Substituting (2) into (5) and adding a vector of disturbance terms $e_{t}$, we obtain the following model $^{1}$ :

$$
w_{t}-w_{t-1}=\Phi B\left(x_{t}-x_{t-1}\right)+\Psi\left(B x_{t-1}-w_{t-1}\right)+e_{t}
$$

Because of the limited amount of data available, however, it appears to be impossible to estimate (6) without imposing restrictions on the matrices of lag parameters $\Phi$ and $\Psi$. The most natural way of imposing restrictions would probably be to suppose $\Phi$ and $\Psi$ to be diagonal. In a similar way as in Berndt and Savin (1975), however, it can be shown that the additivity constraint implies that $\Phi$ and $\Psi$ have to meet the following requirements:

$$
\iota_{n}^{\prime} \Phi=\varphi \iota_{n}^{\prime} \quad \iota_{n}^{\prime} \Psi=\psi \iota_{n}^{\prime} \quad \varphi, \psi \in R
$$

where $\iota_{n}$ denotes the summation vector, i.e. the vector with all elements equal to one. Therefore, we shall assume that each share equation obeys the same adjustment process, i.e. we shall replace the matrices $\Phi$ and $\Psi$ by the scalars $\varphi$ and $\psi$. This leads to the following model:

$$
w_{t}-w_{t-1}=\varphi B\left(x_{t}-x_{t-1}\right)+\psi\left(B x_{t-1}-w_{t-1}\right)+e_{t}
$$

As usual, we shall assume the vectors of disturbance terms $e_{t}$ to be independently and identically distributed over time according to a normal distribution with zero mean and contemporaneous covariance matrix $\Sigma_{n}$, i.e.

$$
e_{t} \sim \operatorname{IN}\left(0, \Sigma_{n}\right)
$$

Because of the additivity constraint, however, it holds true that $\iota_{n}^{\prime} e_{t}=0$, implying that $\Sigma_{n}$ is singular. Barten (1969), however, has shown that this singularity can easily be handled by deleting an arbitrary share equation. Deleting without any loss of generality the last one, we arrive at the following most general form of the model that will be estimated in this paper ${ }^{2}$ :

$$
w_{t}^{(n)}-w_{t-1}^{(n)}=\varphi B^{(n)}\left(x_{t}-x_{t-1}\right)+\psi\left(B^{(n)} x_{t-1}-w_{t-1}^{(n)}\right)+e_{t}^{(n)}
$$

where the superscript $(n)$ denotes that the $n$-th row or $n$-th element has been deleted ${ }^{3}$.

In addition to the model as specified in (9), we shall also consider the following three specifications which are nested into (9):

(a) the partial adjustment $(P A)$ model, which applies when

$$
\varphi=\psi \neq 1
$$

\footnotetext{
${ }^{1}$ In (6) the tilde on the vector $x_{t}$ has been removed because we approximate the equilibrium shares in the Stone-index by their actual values, i.e. we replace $\tilde{P}_{t}=\exp \left\{\sum_{j=1}^{n} \tilde{w}_{t, j} \ln p_{t, j}\right\}$ by $P_{t}=\exp \left\{\sum_{j=1}^{n} w_{t, j} \ln p_{t, j}\right\}$.

${ }^{2}$ Note that the first element of $x_{t}-x_{t-1}$ always equals zero, but this does not cause any problem in the estimation procedure.

${ }^{3}$ Estimates of the parameters of the last share equation are obtained from the additivity constraints as specified in (3).
} 
(b) the first-order autoregressive (AR) model, which is obtained when

$$
\varphi=1, \quad \psi \neq 1
$$

(c) the static (STAT) model, which results when

$$
\varphi=\psi=1
$$

\subsection{Specification of the covariance matrix}

In view of the limited amount of data available, it will be helpful to impose restrictions on the specification of the contemporaneous covariance matrix as well. To that end we shall use a restricted specification which has been introduced by de Boer and Harkema (1997). Letting $\operatorname{diag}\{z\}$ denote a diagonal matrix with the vector $z$ on the main diagonal, the specification reads as follows:

$$
\Sigma_{n}=\operatorname{diag}\left\{\delta_{n}\right\}-d^{-1} \delta_{n} \delta_{n}^{\prime}
$$

with

$$
\delta_{n}^{\prime}=\left[d_{1} \cdots d_{n}\right] \quad \text { and } \quad d=\sum_{i=1}^{n} d_{i}
$$

De Boer and Harkema show that $\Sigma_{n}$ as specified in (10) will be positive semi-definite of rank $(n-1)$ if and only if either all $d_{i}$ 's are positive or at most one $d_{i}$ is negative with $d$ being negative as well. In the former case, the variance of each share equation is smaller than the sum of the variances of the remaining share equation, while in the latter case the variance of the share equation that corresponds with the negative $d_{i}$ is larger than the sum of the variances of the remaining share equations.

Several restricted specifications of the contemporaneous covariance matrix that frequently have been used in applied research are nested into (10). Examples of such specifications are the following:

$$
\Sigma_{n}=\sigma^{2}\left[\operatorname{diag}\left\{\iota_{n}\right\}-n^{-1} \iota_{n} \iota_{n}^{\prime}\right]
$$

where $\iota_{n}$ denotes the summation vector, i.e. the vector with all elements equal to one. This specification has been used by Solari (1971), Deaton (1975), and Winters (1984a) and is obtained when

$$
\begin{gathered}
d_{i}=\sigma^{2} \quad i=1, \cdots, n \\
\Sigma_{n}=\sigma^{2}\left[\operatorname{diag}\left\{w_{n}\right\}-w_{n} w_{n}^{\prime}\right]
\end{gathered}
$$

where $w_{n}^{\prime}=\left[\bar{w}_{1} \cdots \bar{w}_{n}\right]$ with $\bar{w}_{i}$ denoting the average share of country $i$ over the sample period. This specification has been used by Deaton (1975) and applies when

$$
d_{i}=\sigma^{2} \bar{w}_{i} \quad i=1, \cdots, n
$$

and

$$
\Sigma_{n}=\left[\begin{array}{cc}
\operatorname{diag}\{\sigma\} & -\sigma \\
-\sigma^{\prime} & \iota_{n-1}^{\prime} \sigma
\end{array}\right]
$$

where $\sigma^{\prime}=\left[\sigma_{1}^{2} \cdots \sigma_{n-1}^{2}\right]$. This specification has been used by Winters (1984b) and is obtained for

$$
\left|d_{n}\right| \rightarrow \infty \quad \text { and } \quad d_{i}=\sigma_{i}^{2} \quad i=1, \cdots, n-1
$$


The advantage of the covariance matrix as specified in (10) is that it can handle cases in which the variance of one of the share equations is larger than the sum of the variances of the remaining share equations. In the present study, the share of domestic supplies in total supplies is far larger than the other shares. Therefore, it may be expected that its variance will also be larger than the variances of the other shares, and it even seems not unreasonable to presume that its variance will be larger than the sum of the variances of the other shares. Obviously, this case cannot be handled by the other restricted specifications.

\section{Statistical analysis}

\subsection{The estimation procedure}

The models have been estimated by means of the method of maximum likelihood (ML). As regards the model parameters, it should be noted that the model is linear with respect to the elements of the matrix $B^{(n)}$ for given values of the lag parameters $\varphi$ and $\psi$, and vice versa. This property may successfully be used to devise an iterative procedure for the computation of the ML estimators. As regards the elements of the covariance matrix $\Sigma_{n}$, de Boer and Harkema (1997) have shown that the ML estimators $\hat{d}_{i}$ for the parameters $d_{i}(i=1, \cdots, n)$ can be obtained by solving the following system of equations ${ }^{4}$ :

$$
\hat{d}_{i}-\frac{\hat{d}_{i}^{2}}{\hat{d}}=\frac{1}{T-1} \sum_{t=2}^{T} \hat{e}_{t i}^{2} \quad i=1, \cdots, n
$$

where $\hat{e}_{t i}(i=1, \cdots, n-1)$ denotes the residual of the $i$-th share equation at time $t$ and $\hat{e}_{t n}=-\sum_{i=1}^{n-1} \hat{e}_{t i}$. Apart from one special case that occurs with probability zero, the system of equations (11) can be solved in a unique way by means of a one-dimensional search procedure that works very quickly. The algorithm is described in de Boer and Harkema (1997).

The above considerations give rise to the following iterative procedure for the computation of the ML estimators:

(i) choose initial values for $\varphi, \psi$, and the $d_{i}$ 's, for example, $\hat{\varphi}^{(0)}=\hat{\psi}^{(0)}=1$ and $\hat{d}_{i}^{(0)}=1$ $(i=1, \cdots, n)$

(ii) calculate a first-round estimate $\hat{B}^{(1)}$ for $B^{(n)}$, given $\hat{\varphi}^{(0)}, \hat{\psi}^{(0)}$, and the $\hat{d}_{i}^{(0)}$ 's;

(iii) calculate first-round estimates $\hat{\varphi}^{(1)}$ and $\hat{\psi}^{(1)}$ for $\varphi$ and $\psi$, given $\hat{B}^{(1)}$ and the $\hat{d}_{i}^{(0)}$ 's;

(iv) compute first-round residuals $\hat{e}_{t i}^{(1)}(i=1, \cdots, n-1)$, given $\hat{B}^{(1)}, \hat{\varphi}^{(1)}$, and $\hat{\psi}^{(1)}$, according to (9) and first-round residuals $\hat{e}_{t n}^{(1)}$ according to $\hat{e}_{t n}^{(1)}=-\sum_{i=1}^{n-1} \hat{e}_{t i}^{(1)}$;

(v) calculate first-round estimates $\hat{d}_{i}^{(1)}$, given the residuals $\hat{e}_{t i}^{(1)}$, by solving (11);

(vi) repeat steps (ii), (iii), (iv), and (v) until convergence.

As shown by Oberhofer and Kmenta (1974), the above procedure actually converges to a maximum of the likelihood function. As a final remark, it should be noted that the symmetry-constrained version of the model can easily be handled by estimating the model under an appropriate set of linear restrictions on the elements of the matrix $B^{(n)}$.

\footnotetext{
${ }^{4}$ Note that the variances are estimated without imposing any restriction; the restrictions are imposed on the estimates of the covariances.
} 


\subsection{Model selection and a test for structural break}

In order to select a model, we shall adopt the Normalised Akaike Information Criterion (NAIC) as a selection rule because of the following two reasons: (i) it is able to choose between models that are nonnested such as, in the present case, the AR and PA models; (ii) it is able to choose between models which are estimated using different numbers of observations such as, in the present case, the STAT model which is estimated with the first observation included and the dynamic models which are estimated conditional on the first observation. The NAIC reads as follows (see e.g. Stewart (1991), p. 236):

$$
\mathrm{NAIC}=(-2 \ln \hat{L}+2 k) / T
$$

where $\ln \hat{L}$ denotes the natural logarithm of the likelihood function evaluated at its maximum, $k$ represents the number of parameters in the model, and $T$ is the number of observations in the sample. According to this selection rule, we choose the model for which NAIC is a minimum.

The test for structural break is based on the differences between the realized values of the shares in the post-integration period and the shares which are predicted for the post-integration period by the model for the pre- integration period. Weighing the squares and cross-products of these differences in an appropriate way, it can be shown that the resulting test statistic is asymptotically distributed according to a chi-square distribution with $h(n-1)$ degrees of freedom, where $h$ denotes the number of periods predicted ahead. As the derivations are rather involved, a detailed discussion of the test statistic is relegated to the Appendix.

\section{Empirical results}

\subsection{Sources and data}

We do not model Spain's entire imports since this would require dealing with more than a hundred countries, but we aim at covering between 75 and $80 \%$ of total imports. In Table 1 we present the foreign suppliers and we add domestic supplies of manufactures as eighth source. In Table 1 we also present the average shares of the sources over the period between the second oil shock and the accession to the EU (1979-1985) and over the post-integration period (1986-1992). We observe that the shares changed considerably after Spain's accession.

Table 1. Sources and average shares (percentages) 


\begin{tabular}{llrr}
\hline & \multicolumn{1}{c}{ Source } & $1979-1985$ & $1986-1992$ \\
\hline 1. & Germany & 2.37 & 4.51 \\
2. & France & 2.02 & 3.61 \\
3. & Italy & 1.11 & 2.53 \\
4. & Other EU (Belgium/Luxemburg, & 2.28 & 4.30 \\
& Denmark, Greece, Ireland, & & \\
The Netherlands, Portugal, UK) & & \\
\cline { 2 - 3 } & & \\
& EU & & \\
5. Japan & 0.78 & 14.95 \\
6. EFTA (Norway, Sweden, & 0.92 & 1.31 \\
& Austria and Switzerland) & & \\
7. USA and Canada & 2.00 & 2.13 \\
8. Spain & 88.55 & 80.28 \\
\cline { 2 - 3 } & & 100.00 & 100.00 \\
\hline
\end{tabular}

As regards the data, we dispose of a sample of 29 annual observations which relate to the period 1964-1992. The first 22 observations will be used to estimate a model for the pre-integration period, while the last 7 observations are set aside to test for structural break and to assess the impact of the break on the shares of Spain and its trading partners in total supplies. The data are measured as follows.

\section{Foreign supplies and foreign prices}

Imports: imports of manufactures in current prices of the 17 countries were collected from various editions of the "Estadísticas del Comercio Exterior de España" published by the "Dirección General de Aduanas". They have been converted into US dollars by using the conversion factors (US cents per peseta) for imports, published by the United Nations in the International Trade Statistics, Vol. 1, Trade by Country.

Import prices: we have used index numbers of export unit values (in US dollars) collected from various editions of the Yearbooks and Monthly Bulletins of Statistics published by the United Nations. Tariffs: when comparing domestic and foreign variables (prices or supplies) account must also be taken of non- discriminatory tariffs. For the period 1964-1986, only data on total revenues of tariffs from all imports of manufactures (including sources not listed in Table 1) are available so that all revenues were treated as non-discriminatory. Total revenues were divided by total imports of manufactures to yield an average tariff rate and this was used to arrive at tariff-inclusive prices and tariff-inclusive imports. The total revenues of tariffs have been supplied to us by the "Dirección General de Aduanas". For the period 1987-1992 the "Dirección General de Aduanas" supplied us with the split of total tariff revenues over common external tariff revenues (paid by non-EU members) and Spanish external tariff revenues (paid by all countries). Allocating the Spanish external tariff revenues to EU-members and non-EU countries according to their shares in total imports, we were able to calculate separate estimates of the average tariff rate for EU-members and the average tariff rate for non-EU countries.

Domestic supplies and domestic prices

Exports of manufactures and gross value added at factor costs (both in current prices) were collected from the "Contabilidad Nacional" published by the "Instituto Nacional de Estadistica". Exports 
were converted into US dollars by means of the conversion factors (US cents per peseta) for exports, published by the United Nations in the International Trade Statistics, Vol. 1 and gross value added by means of the annual average exchange rates taken from AMECO, a data bank of the European Commission. From Eurostat's VISA data bank it followed that over the period 1980-1992 gross value added in current prices as a percentage of domestic production in current prices was quite stable and amounted to about 33\%. We used this percentage over the whole sample period (1964-1992) in order to estimate domestic production in current prices. Subtracting exports of manufactures from domestic production, we arrived at domestic supplies in current prices. In addition, we found that price series of gross value added and of domestic production in VISA showed a quite similar behaviour in terms of growth rates over the period 1980-1992. Therefore, we use the gross value added price series as a proxy to the true domestic production price series over the whole period 1964-1992 to obtain domestic production in constant prices. We converted it into US dollars by means of the annual average exchange rate for 1980, taken from AMECO. Deflating exports by means of the export unit value of Spain (US cents per peseta) published by the United Nations in the International Trade Statistics, Vol. 1 and subtracting it from domestic production in constant prices, we arrived at domestic supplies in constant prices. Dividing domestic supplies in current prices by domestic supplies in constant prices yields the domestic price series for manufactures. Finally, we transformed all series of explanatory variables (total supplies and prices) into index numbers which take the value 1 in the base year 1980 .

\subsection{Estimation results}

In Table 2, we present the values of the NAIC for the various dynamic specifications of the homogeneityand symmetry-constrained versions of the AID-System.

Table 2. Values of the Normalized Akaike Information Criterion

\begin{tabular}{lcc}
\hline & Homogeneity & Symmetry \\
\hline STAT & -70.45 & -70.17 \\
AR & -72.98 & -71.51 \\
PA & -72.97 & -72.27 \\
ECM & -73.03 & -72.19 \\
\hline
\end{tabular}

As this selection rule chooses the model for which NAIC is a minimum, we select the homogeneityconstrained version of the AID-System with a dynamic specification according to the error correction mechanism. So, all empirical results in the remaining part of the paper pertain to this model.

In Table 3, we present the estimates of the parameters of the AID-System as well as the implied long-run expenditure and own price elasticities (evaluated at the average shares over the period 1979-1985), the estimates of the two parameters describing the error correction mechanism, and the estimates of the parameters of the covariance matrix. The corresponding $t$-values, which are based on the maximum likelihood estimates of the standard errors, are shown between parentheses. As is well-known, however, maximum likelihood estimates may grossly underestimate the true standard errors when the number of observations is small as compared with the number of parameters to be estimated. As a consequence, some correction for degrees of freedom seems to be warranted in order to obtain a fair picture of the significance of the parameter estimates. Neglecting the lag parameters, the number of parameters to be estimated equals $(n+1)$ per equation. Therefore, we shall multiply all estimated standard errors by a factor $\{T /(T-n-1)\}^{\frac{1}{2}}$ or, in the present case, $(21 / 12)^{\frac{1}{2}}$. Using the "two-sigma" rule of thumb to judge the significance of the parameter estimates, a parameter estimate is then considered to be significantly different from zero, when its reported $t$-value exceeds 2.6 in absolute value. 
Table 3. Estimation results for the ECM specification of the homogeneity-constrained AID-System*

\begin{tabular}{|c|c|c|c|c|c|c|c|c|}
\hline & Ger & Fra & $\overline{\text { Ita }}$ & Other EU & Jap & EFTA & USA+Can & Spain \\
\hline \multirow[t]{2}{*}{$\alpha_{i}$} & 0.021 & 0.018 & 0.011 & 0.020 & 0.005 & 0.009 & 0.021 & 0.895 \\
\hline & (10.204) & $(23.492)$ & (13.213) & (14.456) & (7.799) & (10.122) & (9.410) & (129.590) \\
\hline \multirow[t]{2}{*}{$\beta_{i}$} & 0.025 & 0.024 & 0.006 & 0.022 & 0.001 & 0.001 & 0.000 & -0.078 \\
\hline & $(2.747)$ & $(7.185)$ & (1.729) & $(3.468)$ & $(0.334)$ & $(0.147)$ & $(-0.028)$ & $(-2.664)$ \\
\hline \multirow[t]{2}{*}{$\gamma i 1$} & 0.005 & 0.009 & -0.003 & -0.013 & 0.012 & -0.001 & -0.028 & 0.020 \\
\hline & $(0.254)$ & (1.176) & $(-0.402)$ & $(-0.864)$ & (1.664) & $(-0.158)$ & $(-1.244)$ & $(0.281)$ \\
\hline \multirow{2}{*}{$\gamma_{i 2}$} & 0.038 & 0.023 & -0.004 & 0.051 & -0.024 & 0.002 & -0.004 & -0.083 \\
\hline & (1.549) & $(2.577)$ & $(-0.380)$ & (2.911) & $(-2.521)$ & $(0.190)$ & $(-0.136)$ & $(-1.013)$ \\
\hline \multirow{2}{*}{$\gamma_{i 3}$} & 0.002 & -0.004 & 0.010 & 0.013 & -0.006 & 0.004 & 0.011 & -0.031 \\
\hline & $(0.147)$ & $(-0.685)$ & (1.699) & (1.305) & $(-1.320)$ & $(0.753)$ & $(0.744)$ & $(-0.651)$ \\
\hline \multirow[t]{2}{*}{$\gamma_{i 4}$} & 0.011 & 0.029 & 0.011 & 0.011 & -0.004 & 0.007 & -0.019 & -0.045 \\
\hline & $(0.485)$ & $(3.356)$ & (1.198) & $(0.721)$ & $(-0.497)$ & $(0.727)$ & $(-0.790)$ & $(-0.613)$ \\
\hline \multirow[t]{2}{*}{$\gamma_{i 5}$} & -0.013 & -0.018 & -0.009 & -0.006 & -0.012 & 0.000 & -0.008 & 0.066 \\
\hline & $(-1.089)$ & $(-3.643)$ & $(-1.672)$ & $(-0.711)$ & $(-2.806)$ & $(0.052)$ & $(-0.608)$ & $(1.584)$ \\
\hline \multirow[t]{2}{*}{$\gamma_{i 6}$} & -0.088 & -0.072 & -0.015 & -0.071 & 0.021 & -0.004 & 0.026 & 0.202 \\
\hline & $(-2.512)$ & $(-5.209)$ & $(-1.074)$ & $(-2.940)$ & (1.639) & $(-0.308)$ & $(0.709)$ & (1.773) \\
\hline \multirow[t]{2}{*}{$\gamma_{i 7}$} & 0.028 & 0.024 & 0.006 & 0.015 & 0.016 & 0.001 & 0.009 & -0.099 \\
\hline & $(2.956)$ & (6.308) & (1.637) & $(2.332)$ & $(4.873)$ & $(0.310)$ & $(0.899)$ & $(-3.152)$ \\
\hline \multirow[t]{2}{*}{$\gamma_{i 8}$} & 0.017 & 0.009 & 0.004 & 0.000 & -0.003 & -0.009 & 0.012 & -0.031 \\
\hline & (1.047) & $(1.525)$ & $(0.573)$ & $(0.014)$ & $(-0.523)$ & $(-1.308)$ & $(0.709)$ & $(-0.569)$ \\
\hline exp. & 2.037 & 2.205 & 1.554 & 1.968 & 1.154 & 1.058 & 0.987 & 0.911 \\
\hline elas. & $(5.395)$ & (13.147) & $(4.849)$ & (7.048) & $(2.505)$ & (2.698) & $(2.085)$ & (27.369) \\
\hline price & -0.802 & 0.122 & -0.107 & -0.542 & -2.613 & -1.470 & -0.555 & -0.956 \\
\hline elas. & $(-0.914)$ & $(0.275)$ & $(-0.203)$ & $(-0.813)$ & $(-4.548)$ & $(-0.964)$ & $(-1.128)$ & $(-12.385)$ \\
\hline \multirow[t]{2}{*}{$\varphi$} & 0.755 & & & & & & & \\
\hline & $(7.550)$ & & & & & & & \\
\hline \multirow[t]{2}{*}{$\psi$} & 0.467 & & & & & & & \\
\hline & $(6.311)$ & & & & & & & \\
\hline$d_{i}$ & $1.00 \mathrm{E}-06$ & $2.60 \mathrm{E}-07$ & $3.10 \mathrm{E}-07$ & $6.60 \mathrm{E}-07$ & $2.40 \mathrm{E}-07$ & $3.20 \mathrm{E}-07$ & $1.10 \mathrm{E}-06$ & $-4.50 \mathrm{E}-06$ \\
\hline
\end{tabular}

* The numbers between parentheses indicate t-values.

Looking at the parameter estimates in Table 3, let us first consider the estimates of the lag parameters $\varphi$ and $\psi$. Using a one-tailed test at a size of $5 \%$, it turns out that the estimates for $\varphi$ and $\psi$ are significantly different from both zero and unity, which supports our claim that a dynamic specification is necessary in order to provide for an adequate explanation of the data.

With respect to the parameters of the AID-System, it appears that the estimates of the constant terms are all significantly different from zero and practically equal to the values of the average shares over the period 1979-1985 as reported in Table 1. This could be expected, because we measure all explanatory variables by means of index numbers which take the value 1 in the base year 1980. From (1), it then follows that the constant terms can be interpreted as the long-run equilibrium values of the shares in the base year 1980. As regards the parameters which correspond to real demand for manufactures, it appears that there are 4 estimates (out of 8) which are significantly different from zero, viz., the estimates for Germany, France, Other EU, and Spain, with those for Germany, France, and Other EU being positive and the one for Spain being negative. As for the parameters which correspond to the price terms, it turns out that there are 10 estimates (out of 64) which are significantly different from zero. As regards the parameters which correspond to the own prices, it appears that the estimate for Japan is the only one which is significantly different from zero.

The long-run expenditure elasticities $\varepsilon_{t i}$ at time $t$ and the long-run uncompensated price elasticities $\eta_{t i j}$ at time $t$ are easily shown to be equal to:

$$
\varepsilon_{t i}=1+\frac{\beta_{i}}{\tilde{w}_{t i}}\left[1-\frac{\partial \ln \tilde{P}_{t}}{\partial \ln M_{t}}\right] \quad i=1, \cdots, n
$$


and

$$
\eta_{t i j}=-\delta_{i j}+\frac{1}{\tilde{w}_{t i}}\left[\gamma_{i j}-\beta_{i} \frac{\partial \ln \tilde{P}_{t}}{\partial \ln p_{t j}}\right] \quad i, j=1, \cdots, n
$$

where $\delta_{i j}$ denotes the Kronecker delta, i.e. $\delta_{i j}=1$ for $j=i$ and $\delta_{i j}=0$ for $j \neq i$. These expressions can considerably be simplified by assuming that the equilibrium shares $\tilde{w}_{t i}$ are constant over time, i.e. $\tilde{w}_{t i}=\tilde{w}_{i}$, and hence independent of $\ln M_{t}$ and $\ln p_{t j}(j=1, \cdots, n)^{5}$. On this assumption, the long-run expenditure elasticities $\varepsilon_{i}$ and the long-run uncompensated own price elasticities $\eta_{i i}$ are given by:

$$
\varepsilon_{i}=1+\frac{\beta_{i}}{\tilde{w}_{i}} \quad i=1, \cdots, n
$$

and

$$
\eta_{i i}=-1-\beta_{i}+\frac{\gamma_{i i}}{\tilde{w}_{i}} \quad i=1, \cdots, n
$$

The elasticities which are shown in Table 3, are calculated by replacing the model parameters by their estimates and $\tilde{w}_{i}$ by its average share over the period 1979-1985.

As regards the expenditure elasticities, it turns out that the estimates of the elasticities for domestic supplies and imports from USA + Canada are smaller than 1, whereas those for the remaining sources are larger than 1. Using a one-tailed test at a size of $5 \%$ and taking into account the correction for degrees of freedom introduced earlier, it appears that the elasticity of domestic supplies is significantly smaller than 1, while the elasticities of imports from Germany, France, and Other EU are significantly larger than 1 .

As for the own price elasticities, it turns out that the estimates of all elasticities are negative apart from the one for France, which is positive but not significantly different from zero. Using once again a one-tailed test at a size of $5 \%$ and taking into account the correction for degrees of freedom introduced earlier, it appears that the elasticity for Japan is significantly smaller than -1, Spain's elasticity is significantly smaller than zero, but not significantly different from -1, the elasticity for France is significantly larger than -1, but not significantly different from zero, while the elasticities for all other sources are neither significantly different from zero, nor from -1 .

Looking at the results for Spain, it is quite possible that the spectacular increase in real demand and the relatively large inflation rate have contributed to a certain extent to the sharp decline in the share of domestic supplies in total supplies, but it seems very unlikely that these factors can provide for a full explanation of this phenomenon.

As a final remark with respect to Table 3 , it should be noted that the estimate for $d_{n}$, the parameter of the covariance matrix which pertains to Spain, is negative. As said before, this could be expected because the share of domestic supplies in total supplies is far larger than the other shares.

\subsection{Test for structural break and impact assessment}

The value of the test statistic for structural break as described in Section 3.2 and discussed in the Appendix, turned out to be equal to 557. As the critical value of a chi-square distributed random variable with $h(n-1)=49$ degrees of freedom equals 126.15 at a size of $10^{-6 \%}$, it follows that the null

\footnotetext{
${ }^{5}$ Strictly speaking, we should also take into account the derivatives with respect to $\ln M_{t}$ and $\ln p_{t j}$ of the equilibrium shares $\tilde{w}_{t i}$ in $\ln \tilde{P}_{t}$ in calculating the elasticities. Green and Alston (1990) have shown that this leads to a linear equation system from which the elasticities may be solved. Their results show that the differences between the correct expressions and the ones given by (14) and (15) are negligible. Therefore, we shall stick to the assumption that the equilibrium shares are constant over time and use (14) and (15) in order to calculate the elasticities.
} 
hypothesis of no structural break is strongly rejected. So, it is evident that there has been a structural break after Spain's accession to the EU. In our model we have taken account of the increase in real demand and the inflation differentials between Spain and its trading partners, so that the structural break has to be attributed to an important explanatory variable that has been neglected. It seems plausible to presume that that important neglected variable represents Spain's entry into the EU.

Having established the occurrence of a structural break, it is also interesting to assess the impact of the break on the shares of Spain and its trading partners in total supplies. To that purpose, we shall make use of the method of residual imputation. This method assesses the impact by taking the differences between the realized shares in the post-integration period and the shares which are predicted for the post-integration period using the model that has been estimated for the pre-integration period. The results are shown in Table 4. In the upper half of Table 4, we present the shares which are realized in 1985, the year before Spain's entry into the EU, and in the post-integration period 1986-1992. In the lower half, we present the impact of the break, i.e. the differences between the realized shares and the predicted shares.

Looking at the realized shares, it appears that Spain's share in its home market for manufactures continuously declined during the post-integration period from $85.94 \%$ in 1985 to $76.71 \%$ in 1992 . The main beneficiaries of this decline are, as could be expected, the members of the EU, whose joint share almost doubled from $9.90 \%$ in 1985 to $18.14 \%$ in 1992 . The shares of Japan and the EFTA showed a more modest growth of 0.66 and $0.35 \%$ respectively, while the share of the USA and Canada remained practically constant.

Looking at the impact values, it turns out that the structural break led to trade creation with all importing countries apart from the USA and Canada. In addition, it appears that the impacts of the break in 1992 are of the same order of magnitude as the changes in the realized shares during the entire post-integration period. Apparently, the changes in the realized shares are not so much due to the changes in real demand, tariff rates, and prices, but rather to the gradual disappearance of the strong protection (quotas and similar measures) that existed before Spain's accession to the EU as well as the effects of the Single Market Program ${ }^{6}$ (SMP) that were felt well before the official start of the Single Market in 1993.

\footnotetext{
${ }^{6}$ The SMP, launched in 1986 as a working plan, is a set of legislative measures aimed at removing all kinds of unnecessary non-tariff barriers, such as the elimination of border controls, the opening-up of public procurement, and the application of the mutual recognition principle.
} 
Table 4. Realized values and impact in percentages

\begin{tabular}{ccccccccc}
\hline Years & Ger & Fra & Ita & Other EU & Jap & EFTA & USA+Can & Spain \\
\hline & & & \multicolumn{7}{c}{ realized shares } \\
1985 & 3.10 & 2.50 & 1.30 & 3.00 & 0.81 & 1.07 & 2.28 & 85.94 \\
1986 & 3.67 & 2.58 & 1.72 & 3.19 & 1.22 & 1.06 & 1.81 & 84.75 \\
1987 & 4.26 & 3.02 & 2.25 & 3.78 & 1.23 & 1.21 & 1.90 & 82.36 \\
1988 & 4.27 & 3.24 & 2.45 & 4.13 & 1.30 & 1.32 & 2.07 & 81.22 \\
1989 & 4.54 & 3.62 & 2.70 & 4.33 & 1.31 & 1.43 & 2.37 & 79.71 \\
1990 & 4.69 & 3.89 & 2.78 & 4.60 & 1.24 & 1.47 & 2.20 & 79.14 \\
1991 & 4.90 & 4.24 & 2.87 & 4.82 & 1.38 & 1.40 & 2.26 & 78.15 \\
1992 & 5.23 & 4.71 & 2.95 & 5.25 & 1.47 & 1.42 & 2.26 & 76.71 \\
& & & & & & & & \\
1986 & 1.04 & 0.45 & 0.52 & 0.66 & 0.39 & 0.06 & -0.36 & -2.76 \\
1987 & 1.87 & 1.08 & 1.10 & 1.50 & 0.39 & 0.25 & -0.21 & -5.98 \\
1988 & 2.08 & 1.44 & 1.33 & 1.98 & 0.47 & 0.39 & -0.06 & -7.61 \\
1989 & 2.23 & 1.71 & 1.52 & 2.11 & 0.53 & 0.51 & 0.17 & -8.78 \\
1990 & 1.99 & 1.67 & 1.53 & 2.16 & 0.44 & 0.60 & -0.06 & -8.34 \\
1991 & 2.05 & 1.90 & 1.45 & 2.19 & 0.68 & 0.54 & -0.19 & -8.62 \\
1992 & 2.40 & 2.40 & 1.56 & 2.73 & 0.77 & 0.61 & -0.19 & -10.28 \\
\hline
\end{tabular}

\section{Conclusion}

In this paper we have investigated whether Spain's accession to the European Union (EU) has caused a structural break in the allocation of total supplies of manufactures over domestic and foreign suppliers. To that purpose we made use of the homogeneity-constrained version of the Almost Ideal Demand System with a dynamic specification according to the error correction mechanism. It turned out that the spectacular growth in total real demand and the large inflation differentials between Spain and its trading partners could not provide an adequate explanation for the continuous decline of Spain's share in total supplies during the post-integration period. The outcome of a formal statistical test confirmed the occurrence of a structural break after Spain's entry into the EU. In order to assess the impact of the break, we calculated the differences between the realized shares in the post-integration period and the shares that were predicted for the post-integration period by the model for the pre-integration period. It appeared that all trading partners profited by Spain's entry into the EU apart from the USA and Canada, with, as could be expected, the members of the EU being the main beneficiaries.

As a final remark, it should be stressed that one should look at the above results with some caution, because it must be admitted that the approach that has been followed contains some weaknesses. First, we treat manufactures as a single homogeneous good, while a less aggregated approach would perhaps be more appropriate. Secondly, the estimation procedure does not take into account possible simultaneity problems, which may arise because of the presence of the Stone index as an explanatory variable. Last, but not least, we should not forget that the analysis is based on a rather limited amount of data. On the other hand, it should be realized that almost every other exercise of this kind will come across similar problems. Moreover, the observed tendencies are so strong, that it seems very implausible that a more appropriate treatment of the points discussed above could bring on major changes in our results. 


\section{REFERENCES}

Barten, A.P. (1969), Maximum likelihood estimation of a complete system of demand equations, European Economic Review, 1, 7-73.

Berndt, E.R. and N.E. Savin (1975), Estimation and hypothesis testing in singular equation systems with autoregressive disturbances, Econometrica, 43, 937-957.

Boer, P.M.C. de and R. Harkema (1997), A new approach to maximum likelihood estimation of sumconstrained linear models in case of undersized samples, Statistica Neerlandica, 51, 72-89.

Davidson, J.E.H., D.F. Hendry, F. Srba and S. Yeo (1978), Econometric modelling of the aggregate time-series relationship between consumers' expenditure and income in the United Kingdom, The Economic Journal, 88, 661-692.

Deaton, A. (1975), Models and projections of demand in post-war Britain, Chapman and Hall, London.

Deaton, A. and J. Muellbauer (1980), An almost ideal demand system, American Economic Review, $70,312-326$.

Green, R. and J.M. Alston (1990), Elasticities in AIDS models, American Journal of Agricultural Economics, 72, 442-445.

Hamilton, J.D. (1994), Time series analysis, Princeton University Press, New Jersey.

Oberhofer, W. and J. Kmenta (1974), A general procedure for obtaining maximum likelihood estimates in generalized regression models, Econometrica, 42, 579-590.

Solari, L. (1971), Théorie des choix et fonctions de demande sémi-agrégées: modèles statiques, Librairie Droz, Genève.

Stewart, J. (1991), Econometrics, Philip Allan, New York.

Winters, L.A. (1984a), Separability and the specification of foreign trade functions, Journal of International Economics, 17, 239-263.

Winters, L.A. (1984b), British imports of manufactures and the common market, Oxford Economic Papers, 36, 103-118. 


\section{A Appendix}

According to $(9)$, the first $(n-1)$ equations of the dynamic homogeneity-constrained AID-System can be written as ${ }^{7}$ :

$$
w_{t}-w_{t-1}=\varphi B\left(x_{t}-x_{t-1}\right)+\psi\left(B x_{t-1}-w_{t-1}\right)+e_{t}
$$

As regards the vectors of disturbance terms $e_{t}$, it follows from (8) that

$$
e_{t} \sim I N\left(0, \Sigma_{n-1}\right)
$$

where $\Sigma_{n-1}$ denotes the covariance matrix which is obtained by deleting the last row and column of $\Sigma_{n}$.

Under the null hypothesis of no structural break, the model for the prediction periods $T+1, \cdots, T+$ $h$, can be written as:

$$
\left[\begin{array}{cccccc}
I_{n-1} & -(1-\psi) I_{n-1} & 0 & \cdots & 0 & 0 \\
0 & I_{n-1} & -(1-\psi) I_{n-1} & \cdots & 0 & 0 \\
\vdots & \vdots & \vdots & & \vdots & \vdots \\
0 & 0 & 0 & \cdots & I_{n-1} & -(1-\psi) I_{n-1} \\
0 & 0 & 0 & \cdots & 0 & I_{n-1}
\end{array}\right]\left[\begin{array}{c}
w_{T+h} \\
w_{T+h-1} \\
\vdots \\
w_{T+2} \\
w_{T+1}
\end{array}\right]=
$$

$$
\left[\begin{array}{cccccccc}
\varphi B & (\psi-\varphi) B & 0 & \cdots & 0 & 0 & 0 & 0 \\
0 & \varphi B & (\psi-\varphi) B & \cdots & 0 & 0 & 0 & 0 \\
\vdots & \vdots & \vdots & & \vdots & \vdots & \vdots & \vdots \\
0 & 0 & 0 & \cdots & \varphi B & (\psi-\varphi) B & 0 & 0 \\
0 & 0 & 0 & \cdots & 0 & \varphi B & (\psi-\varphi) B & (1-\psi) I_{n-1}
\end{array}\right]\left[\begin{array}{c}
x_{T+h} \\
x_{T+h-1} \\
\vdots \\
x_{T+1} \\
x_{T} \\
w_{T}
\end{array}\right]+\left[\begin{array}{c}
e_{T+h} \\
e_{T+h-1} \\
\vdots \\
e_{T+2} \\
e_{T+1}
\end{array}\right]
$$

It is easy to verify that the inverse of the matrix at the left-hand side of the equality sign in (18) is equal to:

$$
J=\left[\begin{array}{cccccc}
I_{n-1} & (1-\psi) I_{n-1} & (1-\psi)^{2} I_{n-1} & \cdots & (1-\psi)^{h-2} I_{n-1} & (1-\psi)^{h-1} I_{n-1} \\
0 & I_{n-1} & (1-\psi) I_{n-1} & \cdots & (1-\psi)^{h-3} I_{n-1} & (1-\psi)^{h-2} I_{n-1} \\
0 & 0 & I_{n-1} & \cdots & (1-\psi)^{h-4} I_{n-1} & (1-\psi)^{h-3} I_{n-1} \\
\vdots & \vdots & \vdots & & \vdots & \vdots \\
0 & 0 & 0 & \cdots & (1-\psi) I_{n-1} & (1-\psi)^{2} I_{n-1} \\
0 & 0 & 0 & \cdots & I_{n-1} & (1-\psi) I_{n-1} \\
0 & 0 & 0 & \cdots & 0 & I_{n-1}
\end{array}\right]
$$

In order to obtain explicit expressions for the vectors of shares in the prediction periods $T+1, \cdots, T+h$, we have to premultiply both sides of (18) with the matrix $J$. To that purpose, we start by considering the vector $w^{*^{\prime}}=\left[w_{T+h}^{*^{\prime}} \cdots w_{T+1}^{*^{\prime}}\right]$, which is the product of the matrix $J$ with the first term in the right-hand side of (18). Defining

$$
z_{T+1}=\varphi x_{T+1}+(\psi-\varphi) x_{T}
$$

\footnotetext{
${ }^{7}$ For reasons of notational convenience, we drop the superscripts $(n)$ in this Appendix.
} 
and

$$
\begin{aligned}
z_{T+i}= & \varphi x_{T+i}+\sum_{j=0}^{i-2}(1-\psi)^{j} \psi(1-\varphi) x_{T+i-1-j}+ \\
& +(1-\psi)^{i-1}(\psi-\varphi) x_{T} \quad i=2, \cdots, h
\end{aligned}
$$

it is straightforward, though rather tedious, to verify that $w_{T+i}^{*}$ is given by

$$
w_{T+i}^{*}=B z_{T+i}+(1-\psi)^{i} w_{T} \quad i=1, \cdots, h
$$

or, applying the vec operator to the matrix $B$, by

$$
w_{T+i}^{*}=\left[z_{T+i}^{\prime} \otimes I_{n-1}\right] \operatorname{vec} B+(1-\psi)^{i} w_{T}
$$

where $\otimes$ denotes the Kronecker product operator.

Stacking the vectors $w_{T+i}^{*}$ one underneath the other, it follows that the vector $w^{*}$ can be written as

$$
w^{*}=Z \operatorname{vec} B+K w_{T}
$$

with

$$
Z=\left[\begin{array}{ccc}
z_{T+h}^{\prime} & \otimes & I_{n-1} \\
& \vdots & \\
z_{T+1}^{\prime} & \otimes & I_{n-1}
\end{array}\right] \text { and } K=\left[\begin{array}{c}
(1-\psi)^{h} I_{n-1} \\
\vdots \\
(1-\psi) I_{n-1}
\end{array}\right]
$$

Multiplying the matrix $J$ with the second term in the right-hand side of (18) and denoting the resulting vector by $v$, it follows from (17) that

$$
v=J\left[\begin{array}{c}
e_{T+h} \\
\vdots \\
e_{T+1}
\end{array}\right] \sim N\left\{0, J\left(I_{h} \otimes \Sigma_{n-1}\right) J^{\prime}\right\}
$$

So, denoting the vector of actual shares in the prediction periods $T+1, \cdots, T+h$ by $w^{\prime}=\left[w_{T+h}^{\prime} \cdots w_{T+1}^{\prime}\right]$, it follows from (23) and (24) that $w$ may be written as

$$
\begin{aligned}
w & =w^{*}+v \\
& =Z \operatorname{vec} B+K w_{T}+v
\end{aligned}
$$

Denoting the vector of predictions for the shares in the periods $T+1, \cdots, T+h$ by $w^{p^{\prime}}=$ $\left[w_{T+h}^{p^{\prime}} \cdots w_{T+1}^{p^{\prime}}\right]$, it follows from $(25)$ that $w^{p}$ is given by

$$
w^{p}=\hat{Z} \operatorname{vec} \hat{B}+\hat{K} w_{T}
$$

where hats indicate that all parameters in $Z$, vec $B$, and $K$ have been replaced by their maximum likelihood estimates.

From (25) and (26), it follows that the vector of prediction errors $w-w^{p}$ is given by

$$
w-w^{p}=w^{*}-w^{p}+v
$$

In order to derive the distribution of the vector of prediction errors, we start by defining the vector of parameters $\theta$ and the vector of maximum likelihood estimates $\hat{\theta}$ according to 


$$
\theta=\left[\begin{array}{c}
\operatorname{vec} B \\
\varphi \\
\psi
\end{array}\right] \text { and } \hat{\theta}=\left[\begin{array}{c}
\operatorname{vec} \hat{B} \\
\hat{\varphi} \\
\hat{\psi}
\end{array}\right]
$$

As $\hat{\theta}$ is a vector of maximum likelihood estimates, it is well-known that the limiting distribution of $\sqrt{T}(\hat{\theta}-\theta)$ for $T$ approaching infinity is given by

$$
\sqrt{T}(\hat{\theta}-\theta) \stackrel{\text { asy }}{\sim} N\{0, V\}
$$

where

$$
V=\lim _{T \rightarrow \infty} T[I(\theta)]^{-1}
$$

with $I(\theta)$ denoting the information matrix with respect to $\theta$.

Let us now first consider the term $w^{*}-w^{p}$ in the right-hand side of (27). Because $w^{*}$ is a differentiable function of $\theta$, it follows from (28) (see e.g. Hamilton (1994), Proposition 7.4) that the limiting distribution of $\sqrt{T}\left(w^{*}-w^{p}\right)$ for $T$ approaching infinity is given by

$$
\sqrt{T}\left(w^{*}-w^{p}\right) \stackrel{\text { asy }}{\sim} N\left\{0, H V H^{\prime}\right\}
$$

where $H$ denotes the matrix of first-order derivatives of $w^{*}$ with respect to $\theta^{\prime}$. From (23) and the expressions in (20) and (21), it is easily verified that the matrix $H$ looks as follows:

$$
H=\left[H_{1}: H_{2}: H_{3}\right]
$$

with

$$
H_{1}=\left[\begin{array}{ccc}
z_{T+h}^{\prime} & \otimes & I_{n-1} \\
& \vdots & \\
z_{T+2}^{\prime} & \otimes & I_{n-1} \\
z_{T+1}^{\prime} & \otimes & I_{n-1}
\end{array}\right] \quad H_{2}=\left[\begin{array}{ccc}
r_{T+h}^{\prime} & \otimes & I_{n-1} \\
& \vdots & \\
r_{T+2}^{\prime} & \otimes & I_{n-1} \\
r_{T+1}^{\prime} & \otimes & I_{n-1}
\end{array}\right] \text { vecB }
$$

and

$$
H_{3}=\left[\begin{array}{ccc}
s_{T+h}^{\prime} & \otimes & I_{n-1} \\
& \vdots & \\
s_{T+2}^{\prime} & \otimes & I_{n-1} \\
s_{T+1}^{\prime} & \otimes & I_{n-1}
\end{array}\right] \quad \operatorname{vec} B-\left[\begin{array}{c}
h(1-\psi)^{h-1} I_{n-1} \\
\vdots \\
2(1-\psi) I_{n-1} \\
I_{n-1}
\end{array}\right] w_{T}
$$

where

$$
\begin{gathered}
r_{T+i}=\frac{\partial z_{T+i}}{\partial \varphi}=x_{T+i}-\sum_{j=0}^{i-2}(1-\psi)^{j} \psi x_{T+i-1-j}-(1-\psi)^{i-1} x_{T} \quad i=2, \cdots, h ; \\
r_{T+1}=\frac{\partial z_{T+1}}{\partial \varphi}=x_{T+1}-x_{T} \\
s_{T+i}=\frac{\partial z_{T+i}}{\partial \psi}=\sum_{j=0}^{i-2}\left[(1-\psi)^{j}(1-\varphi)-j(1-\psi)^{j-1} \psi(1-\varphi)\right] x_{T+i-1-j}+ \\
+\left[(1-\psi)^{i-1}-(i-1)(1-\psi)^{i-2}(\psi-\varphi)\right] x_{T} \quad i=2, \cdots, h ;
\end{gathered}
$$


and

$$
s_{T+1}=\frac{\partial z_{T+1}}{\partial \psi}=x_{T}
$$

From (29), it follows that the distribution of $w^{*}-w^{p}$ may be approximated by

$$
w^{*}-w^{p} \approx N\left\{0, H[I(\theta)]^{-1} H^{\prime}\right\}
$$

for $T$ sufficiently large.

Next, it should be noted that the terms $\left(w^{*}-w^{p}\right)$ and $v$ in $(27)$ are independently distributed. From (24) and (30), it then follows that the distribution of the vector of prediction errors $w-w^{p}$ may be approximated by

$$
w-w^{p} \approx N\left\{0, \hat{H}[I(\hat{\theta})]^{-1} \hat{H}^{\prime}+\hat{J}\left(I_{h} \otimes \hat{\Sigma}_{n-1}\right) \hat{J}^{\prime}\right\}
$$

where hats once again indicate that all unknown parameters have been replaced by their maximum likelihood estimates. Under the null hypothesis of no structural break, the test statistic

$$
S=\left(w-w^{p}\right)^{\prime}\left\{\hat{H}[I(\hat{\theta})]^{-1} \hat{H}^{\prime}+\hat{J}\left(I_{h} \otimes \hat{\Sigma}_{n-1}\right) \hat{J}^{\prime}\right\}^{-1}\left(w-w^{p}\right)
$$

will, therefore, approximately be distributed according to a chi-square distribution with $h(n-1)$ degrees of freedom for $T$ sufficiently large. 